\title{
Donor/recipient enhancement of memory in rat hippocampus
}

\author{
Sam A. Deadwyler ${ }^{1 *}$, Theodore W. Berger ${ }^{2}$, Andrew J. Sweatt ${ }^{1}$, Dong Song ${ }^{2}$, Rosa H. M. Chan ${ }^{2}$, \\ Ioan Opris ${ }^{1}$, Greg A. Gerhardt ${ }^{3}$, Vasilis Z. Marmarelis ${ }^{2}$ and Robert E. Hampson ${ }^{1}$ \\ ${ }^{1}$ Department of Physiology and Pharmacology, Wake Forest School of Medicine, Winston-Salem, NC, USA \\ 2 Department of Biomedical Engineering, University of Southern California, Los Angeles, CA, USA \\ ${ }^{3}$ Department of Neurobiology, Chandler Medical School, University of Kentucky, Lexington, KY, USA
}

Edited by:

Mikhail Lebedev, Duke University, USA

\section{Reviewed by:}

Antonio Pereira, Federal University of Rio Grande do Norte, Brazil Nelson A. M. Lemos, Edmond and Lily Safra International Institute of Neuroscience of Natal, Brazil Mark O. West, Rutgers University, USA

*Correspondence:

Sam A. Deadwyler, Department of Physiology and Pharmacology, Wake Forest University Health Sciences, Medical Center Blvd.

Winston-Salem, NC 27157, USA e-mail: sdeadwyl@wakehealth.edu
The critical role of the mammalian hippocampus in the formation, translation and retrieval of memory has been documented over many decades. There are many theories of how the hippocampus operates to encode events and a precise mechanism was recently identified in rats performing a short-term memory task which demonstrated that successful information encoding was promoted via specific patterns of activity generated within ensembles of hippocampal neurons. In the study presented here, these "representations" were extracted via a customized non-linear multi-input multi-output (MIMO) mathematical model which allowed prediction of successful performance on specific trials within the testing session. A unique feature of this characterization was demonstrated when successful information encoding patterns were derived online from well-trained "donor" animals during difficult long-delay trials and delivered via online electrical stimulation to synchronously tested naïve "recipient" animals never before exposed to the delay feature of the task. By transferring such model-derived trained (donor) animal hippocampal firing patterns via stimulation to coupled naïve recipient animals, their task performance was facilitated in a direct "donor-recipient" manner. This provides the basis for utilizing extracted appropriate neural information from one brain to induce, recover, or enhance memory related processing in the brain of another subject.

Keywords: memory-transfer, rodent, ensemble, non-linear model, electrical stimulation

\section{INTRODUCTION}

To understand the neural basis of memory, several features of the context in which the memories occur and are utilized, and the functional aspects of the brain areas involved, need to be identified and controlled (Hampson et al., 2008; Eichenbaum and Fortin, 2009). In prior studies we achieved both of these important contingencies as well as (1) overcoming possible alternative interpretations of the relationship between recorded hippocampal ensemble activity and the behavioral task in which short-term memory formation is necessary (Deadwyler and Hampson, 2006; Deadwyler et al., 2007), and (2) developing an effective mathematical/operational model for online prediction of CA1 hippocampal cell activity from simultaneously recorded input firing patterns from synaptically connected CA3 neurons (Song et al., 2009; Berger et al., 2011; Hampson et al., 2011). The combination of these approaches was made possible by the chronic recording of neural firing patterns in the above two major hippocampal subfields via specially designed mutineuron recording arrays that allowed simultaneous detection and analysis of behaviorally critical ensemble discharge patterns (Deadwyler and Hampson, 1997; Hampson et al., 1999, 2008). It has been shown that a nonlinear multi-input/multi-output (MIMO) mathematical model provides the mean to translate the above ensemble activity into a format that allows predictions of CA1 firing patterns from CA3 activity required for successful task performance (Marmarelis, 2004; Zanos et al., 2008; Song et al., 2009).

In the following paper we demonstrate a critically important feature of the online extracted firing patterns of hippocampal ensembles by showing how alteration and facilitation of hippocampal function can be employed via direct connection with the same structure in a different "donor" animal performing the same task at the same time. A recent study (Pais-Viera et al., 2013) reported similar brain-to-brain transfer by directly stimulating motor cortex in the brain of a recipient rodent from a different animal. However, we report here the discovery that appropriate neural firing patterns which encode useable memory can be derived online from trained animals, and inserted via electrical stimulation of those same hippocampal regions to animals untrained to perform the memory extended requirement of the delayed-non-match-to-sample (DNMS) task. These findings confirm the functional significance of a previously identified "hippocampal prosthesis" (Berger et al., 2011) shown to repair and/or enhance damaged or disrupted memory processes in the same animal. However, the outcomes of the study described here also indicate ways of using brain systems from non-impaired subjects to impart functional information when and where it did not get formulated in affected subjects, via a donor-recipient paradigm. 


\section{METHODS \\ INFORMATION ENCODING BY HIPPOCAMPAL NEURAL ENSEMBLES DURING PERFORMANCE OF A DELAYED-NON-MATCH-TO-SAMPLE (DNMS) MEMORY TASK}

Recording and analysis of hippocampal mutineuron activity over a number of years in rodents performing a DNMS memory task (cf. Hampson et al., 2008, 2011) provided the basis for application of a non-linear MIMO model to hippocampal neural ensemble firing patterns in the first demonstration of a memory prostheses in rodent brain (Berger et al., 2011; Hampson et al., 2012a,b). This extensively studied DNMS task requires rats to retain the position of a "Sample" lever that is presented and responded to (i.e., sample response: SR) at the start of the trial, over a temporal delay interval of variable duration (1-30s) in order to make a "Nonmatch" response (NR) on the lever in the opposite position when both levers are presented simultaneously at the end of the delay (Figure 1A). During the delay period a nosepoke into a photocell on the wall opposite the levers is required to proceed to the Nonmatch phase. If there is no delay a single nosepoke produces both levers, for delays of increased duration a single nose poke is still the only requirement but animals make multiple nosepokes until the light terminates above the photocell as an indicator of delay termination and both levers are presented on the opposite wall (Figure 1A). Figure 1D (control) shows that DNMS performance accuracy decreases linearly as a direct function of the duration of the interposed delay interval.

\section{NON-LINEAR MULTI-INPUT MULTI-OUTPUT (MIMO) MODEL DETECTION AND PREDICTION OF HIPPOCAMPAL ENSEMBLE MEMORY CODES}

Electrophysiological recording during the DNMS task employs custom designed arrays of microwire $(20 \mu \mathrm{m})$ electrodes implanted bilaterally in the hippocampus in each hemisphere to provide single neuron firing data from 8 pairs of aligned CA3CA1 probes arranged at $200 \mu \mathrm{m}$ intervals along the longitudinal axis in the dorsal hippocampus in rodent brain (Figure 1B). The neural correlates obtained from studies with these techniques in the DNMS task have been utilized in more than 2000 animals with respect to type and amount of behavioral training required for maximal performance in conjunction with extraction of distinct patterns of neural ensemble activity correlated with successful performance (Deadwyler and Hampson, 2006; Hampson et al., $2008,2011)$. In recent studies the trial-by-trial nature of changes in ensemble firing patterns has been described in relation to nonlinear fluctuations associated with successful task performance (Figure 1C), as well as applying the same non-linear model for reversing detrimental actions of drugs on performance (Song et al., 2009; Berger et al., 2011; Marmarelis et al., 2013).

This very precise MIMO non-linear mathematical model (Figure 1C) was employed to determine the "strength" of ensemble SR firing patterns or "codes" formulated specifically on successful (strong code) or error (weak code) DNMS trials (Figures 1C,D) across all delay durations. The application of this model (see Supporting Material) allowed prediction of CA1 neuron firing "output" patterns based on the "input" to the model (CA3 neuron firing) using Laguerre expansions of Volterra Kernels to determine the temporal relationships between spike occurrences recorded in these two areas during the task (Song et al., 2009, 2013; Berger et al., 2012). As shown previously the inputs to the MIMO model were CA3 cell discharges associated exclusively with outputs from simultaneously recorded postsynaptic CA1 cells connected via Schaeffer collateral monosynaptic connections (Witter and Amaral, 2004). Hence, as shown at the lower right in Figure 1C, the MIMO model analysis of CA3 and CA1 spike occurrences associated with critical DNMS task events provided the basis for online "detection" (CA3) and "prediction" of CA1 firing patterns associated with successful (strong code) vs. error (weak code) trials (Berger et al., 2011; Hampson et al., 2011). As a final verification that the MIMO model output could predict behaviorally relevant hippocampal encoded information, online calculations were utilized in a closed loop paradigm in which the detection of strong SR codes (Figure 1C) was used to adjust the difficulty of the same trial via increased or decreased delay duration. In accordance with the strength of SR codes (Figure 1D) performance was either above or below that on trials in which such SR codes were not present (Hampson et al., 2012a,b). This closed loop procedure served as the basis for the next phase in which strong SR code patterns were detected, mimicked and then administered as electrical stimulation, which is described next.

\section{EXTERNAL INSERTION OF MIMO DERIVED FIRING PATTERNS VIA ELECTRICAL STIMULATION OF HIPPOCAMPAL CA1 NEURONS}

The above successful application of the MIMO model provided the unique basis for activating CAl cells if model derived inputs from CA3 neurons were no longer operative (Berger et al., 2011). This was accomplished by transforming the CA1 cell output pattern of the MIMO model into trains of electrical stimulation pulses $(1.0 \mathrm{~ms}$ biphasic $20-100 \mu \mathrm{A})$ and delivering them in real time to the same CA1 electrode locations in the recording arrays via a multichannel stimulator (Figure 2A). CA1 stimulation patterns were therefore similar to the strong code SR firing patterns derived from each animal by the MIMO model from the same CA1 electrodes. Stimulation was delivered online via the inputs detected from CA3 electrodes on the same hippocampal array as shown in Figure 2A for hippocampal implants in both hemispheres. Intensities of stimulus pulses $(20-100 \mu \mathrm{A})$ delivered to CA1 were adjusted to provide indications of extracellular current flow (i.e., local field potentials) at adjacent CA1 electrode locations on the same array (see below). MIMO generated strong code CA1 SR stimulation pulse trains were of $3.0 \mathrm{~s}$ duration and delivered within $50 \mathrm{~ms}$ of the detection of corresponding input patterns recorded in CA3 (see Supporting Material). Since both the pattern and time of application of the strong code CA1 SR stimulation were related directly to the MIMO model detection of corresponding CA3 input firing, it was also possible to deliver CA1 SR stimulation on trials in which "strong code" input patterns were not detected in CA3 recordings. This provided the means to facilitate performance above control levels by delivering strong code CA1 SR stimulation on trials that normally did not generate strong codes in CA3 or CA1 naturally (Berger et al., 2011; Hampson et al., 2012a,b).

The effectiveness of SR CA1 stimulation patterns is shown in Figures 2B,C as marked increases in DNMS task performance 


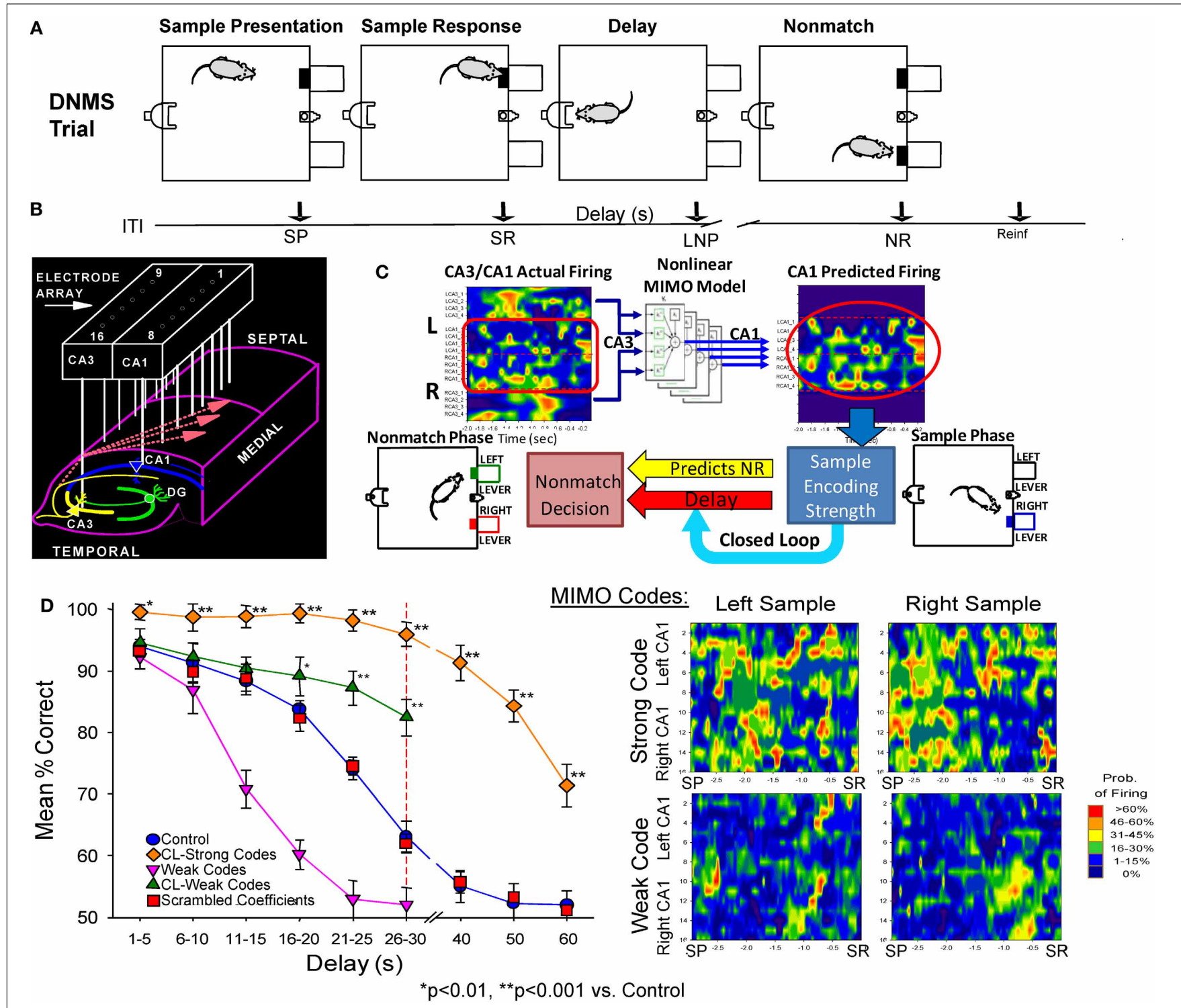

FIGURE 1 | Delayed non-match to sample (DNMS) task, MIMO model and associated hippocampal ensemble activity. (A) DNMS Trial Diagram. Sample lever presentation (SP) and Sample response (SR) are followed by a variable delay interval which required a nosepoke (NP) in a photocell on opposite wall. The Non-match phase began after delay timeout, with both levers presented simultaneously for reward contingent Non-match Response (NR) on the lever opposite the SR position. Correct non-match responses produced $0.2 \mathrm{ml}$ of water delivered to the trough between the levers. Timeline below shows sequence of task phases: ITI-intertrial interval; SP-Sample Lever presentation; SR-Sample response; Delay-Delay interval; LNP-last required nosepoke during Delay; NR-Non-match (decision) response; Reinf.-Delivery of water reward. (B) Hippocampal recording array: two rows of 8 stainless steel $20 \mu \mathrm{m}$ wires positioned longitudinally within hippocampus at $200 \mu \mathrm{m}$ intervals for each electrode pair in CA3 and CA1 cell layers. Arrays were implanted bilaterally in both hippocampi providing a total of 32 indwelling chronic electrodes per animal. (C) Heatmap display (left) showing online array monitored hippocampal ensemble single neuron (actual firing) activity. Low-to-high (blue-to-red) firing rates are indicated at the separate CA3/CA1 locations on the array (B) during the occurrence of the SR (time 0.0 s). Schematic of non-linear MIMO model: Spike trains $X_{1}-X_{8}$ recorded from CA3 electrodes (CA3 input) on the hippocampal array (left) are input to the model and used to predict CA1 firing across the other 8 recording locations shown in the diagram on the right (1-8, predicted CA1) at the time of the SR. The schematic of the non-linear analysis used to construct the CA1 predicted outputs which illustrates estimation of the spatiotemporal relationship between each $\mathrm{CA} 1$ output $(\mathrm{Y})$ and multiple $\mathrm{CA} 3$ inputs $(X)$ modeled via Volterra kernels which are then combined to form the MIMO model for all CA1 locations (see Supplemental Material). The output of the model (right) is then employed to vary the delay interval of the DNMS task on the same trial in a closed loop manner as shown by the diagram below. Lower Right: MIMO Codes: Heatmap displays of MIMO model predicted CA1 firing in both hemispheres during the response on the Sample lever on individual trials during sample presentation (SP and response (SR) for trials both Left and Right sample lever presentation. Strong Codes: MIMO predicted CA1 sample lever firing on successful trials. Weak Codes: MIMO prediction of the same CA1 cell firing on error trials. Firing rates indicated by the scale bar at right. (D) MIMO mediated closed loop control of DNMS performance (mean \pm s.e.m. \% correct) summed over all animals, $n=15$ ). Trials in which strong (diamonds) and weak (triangles) SR codes occurred are plotted as a function of length of delay, shown compared to Control performance on trials not sorted by code strength. Performance on trials with extended delays $(40,50$, or $60 \mathrm{~s}$, vertical dashed line) was significantly higher than on trials with the same delays (Control, 40-60 s) presented without (Continued) 


\section{FIGURE 1 | Continued}

MIMO Closed Loop regulation $\left[F_{(1,401)}=18.39, p<0.001, * p<0.01\right.$, ${ }^{* *} p<0.001$, Closed Loop vs. Control trials]. DNMS (performance) for trials of $1-30 \mathrm{~s}$ delay (Control) is also shown compared to performance on trials in which only weak SR codes (Weak Codes) occurred $\left[F_{(1,401)}=11.81, p<0.001\right]$. Performance on trials in which the MIMO model coefficients were randomly assigned (i.e., scrambled) to CA1 firing are also shown in the curve for scrambled coefficients (squares) as having no difference from Control performance.

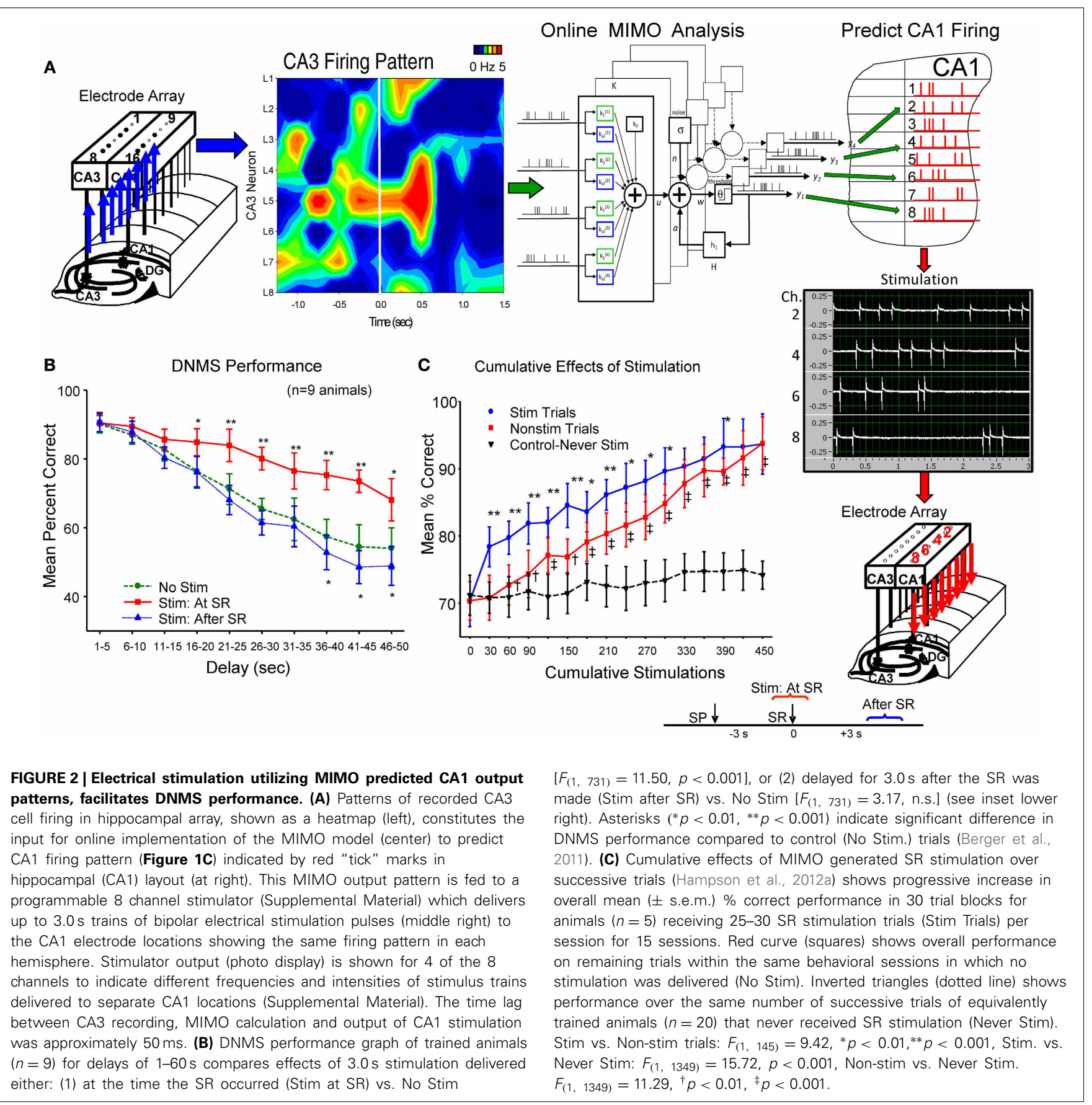

on stimulation trials in comparison to trials in which no stimulation was delivered (No Stim). To control for other possible actions, the specificity of the CA1 stimulation pattern with respect to encoding of the SR was tested directly by delaying delivery of the same stimulation pattern to CA1 until $3.0 \mathrm{~s}$ after the SR which

as shown in Figure 2B (Stim after SR) produced no changes in performance from control (No Stim) levels. Further verification was revealed by comparing trials in which SR CA1 stimulation was generated from different MIMO firing patterns with "scrambled" coefficients between neurons (Figure 1D) which actually 
impaired performance in some cases as shown in other studies (Hampson et al., 2012a). A final test of the similarity of the stimulation patterns to actual CA1 output firing patterns was assessed by repeating the procedure over several sessions and examining the trial-by-trial cumulative effects of continued exposure to MIMO predicted SR CA1 stimulation as shown in Figure 2C (Berger et al., 2011; Hampson et al., 2012b). These procedures verify that functional encoding of the SR could be imposed in subjects performing the DNMS task by matching the MIMO predicted CA1 firing pattern with stimulation pulses delivered within $50 \mathrm{~ms}$ to the same CA1 loci (Figure $2 \mathrm{~A}$ ), which provided encoding of lever position necessary to perform the task successfully across all interposed delay intervals.

\section{A METHOD FOR TRANSFER OF MIMO SR STIMULATION FROM DONOR TO DELAY-NAIVE RECIPIENT ANIMALS}

The above MIMO model SR CA1 stimulation method served as the basis for testing a unique dual animal "donor/recipient" paradigm in which (1) a well-trained "donor rat" performed the DNMS task in one chamber at the same time as (2) a delay-naïve "recipient rat" was tested at the same time in a different chamber in a trial synchronized manner (Figure 3A). The "recipient rat" was not trained to perform the DNMS task over intervening delay intervals $>1.0-3.0 \mathrm{~s}$ (red dotted delay phase in Figure 3B) which was the time it took to make the required nosepoke response on the opposite wall of the chamber to present the Non-match phase (Figure 1A). The imposition of trials with extended delay intervals during the session constituted the first exposure of recipient rats to the task requirement for retention of SR information across increased time intervals (8-16s) in order to correctly select the opposite lever in the Non-match phase (Hampson et al., 2008). Performance in the Sample phase of the task was synchronized between animals by presentation of the Sample lever in the same position at the same time in both chambers to initiate the same trial simultaneously for both animals (Figure 3B). On synchronous trials in which the MIMO model applied to the donor rat CA3 firing patterns, generated a successful strong code SR CA1 pattern (Figure 2A), the stimulus pulses representing that MIMO strong SR code were routed instead to the corresponding CA1 electrodes in the recipient rat (Figure 3A) performing the SR at approximately the same time. For the naïve recipient rat following the delivery of the donor rat strong code SR CA1 stimulation pattern after the SR, an unfamiliar delay interval of 8,12 , or $16 \mathrm{~s}$ was introduced into the trial prior to onset of the Non-match phase. Since routing of SR CA1 stimulation to the recipient rat was determined by concurrent CA3 encoding strength in the hippocampus of the simultaneously performing donor rat, extended delay trials for the recipient rat occurred randomly within the paired sessions. As a control procedure, performance was compared on trials with the same delays administered to recipient rats by donor rat CA3 encoding strength on the same trial but without delivery of SR CA1 stimulation.

\section{RESULTS}

TRANSFER OF MEMORY TO DELAY-NAIVE RECIPIENT ANIMALS BY DELIVERY OF MIMO STIMULATION FROM TRAINED DONOR ANIMALS

The employment of donor rat MIMO model generated SR CA1 stimulation was applied to test whether it was possible to facilitate performance in delay-naïve recipient rats untrained in the delay version of the DNMS task. Results in Figure 4A show the mean $\%$ correct performance of 5 different recipient rats subjected for the first time to 8,12 , and $16 \mathrm{~s}$ interpolated delays with MIMO derived SR CA1 stimulation patterns (Stim) delivered on half the trials from synchronously performing donor rats. Donor rat SR CA1 stimulation allowed recipient rats to perform significantly better than if they did not receive stimulation (No stim) and these levels are compared with their higher performance on trials in which no delay was interspersed $(0.0 \mathrm{~s})$. The overall performance of all recipient is shown in Figure 4B for recipient-stim and recipient-no stim trials in which highly significant improved performance is apparent across all delays. Figure 4B also shows that recipient-stim average performance was significantly below that of fully trained animals that did not receive SR stimulation at those same delays (trained subjects, $n=23$ ).

The physiologically specific nature of the donor rat stimulation was further verified by the fact that performance was facilitated in a delay dependent manner (Figures $4 \mathbf{A}, \mathbf{B}$ ) in the same way that natural performance was affected by the duration of interposed delays during the trial. This was further verified by comparing the effects of MIMO stimulation patterns delivered by different donor rats to the same recipient rat within, as well as, across different behavioral sessions. Figure 5A shows the comparison of performance of the same recipient rat receiving SR stimulation from two different donor rats over similar interposed delay trials. Figure 5A shows the patterns of SR CA1 stimulation delivered by each donor rat (donor rats 1 and 2) on both left and right SR lever trials. Although there were slight differences with respect to the spatiotemporal delivery of CA1 stimulation pulses, the overall patterns related to the time of execution of the SR were highly similar. The graphs to the right in Figure 5A show the average performance of the same recipient rat on similar types of trials with stimulation and non-stimulated extended delays from the same two donor rats. It is clear that stimulation generated by both donor rats on different trials in the same session facilitated performance of the recipient rat in nearly identical fashion. Figure 5B summarizes the performance of all recipient rats $(n=5)$ over the 3 delay intervals for all donor rat $(n=6)$ stimulation (Stim) vs. non-stimulated (Non-Stim) trials compared to trials in which no delay was imposed ( $0 \mathrm{~s})$.

Control manipulations performed to insure that donor rat SR stimulation was the basis for the improved performance of recipient rats included: (1) changing the donor rat stimulation patterns in different ways such as scrambling coefficients (Figure 1A), (2) delivering donor rat stimulation patterns at different times (i.e., $3.0 \mathrm{~s}$ ) after the SR (Figure 2B), and (3) delivery of stimulation based on recipient rat MIMO-extracted SR CA1 patterns on the trials with interpolated delays determined by donor rat encoding patterns (not shown). None of the latter control procedures produced significant increases in recipient rat performance above that exhibited on trials with the same delays and no donor rat stimulation (Figure 4 No Stim, Figure 5B).

\section{NEURAL BASIS FOR MIMO STIMULATION ENHANCED MEMORY}

A major factor that relates to the above demonstration of enhanced memory in the donor as well as delay-naïve recipient rats, is the actual neural basis for the enhancement invoked 


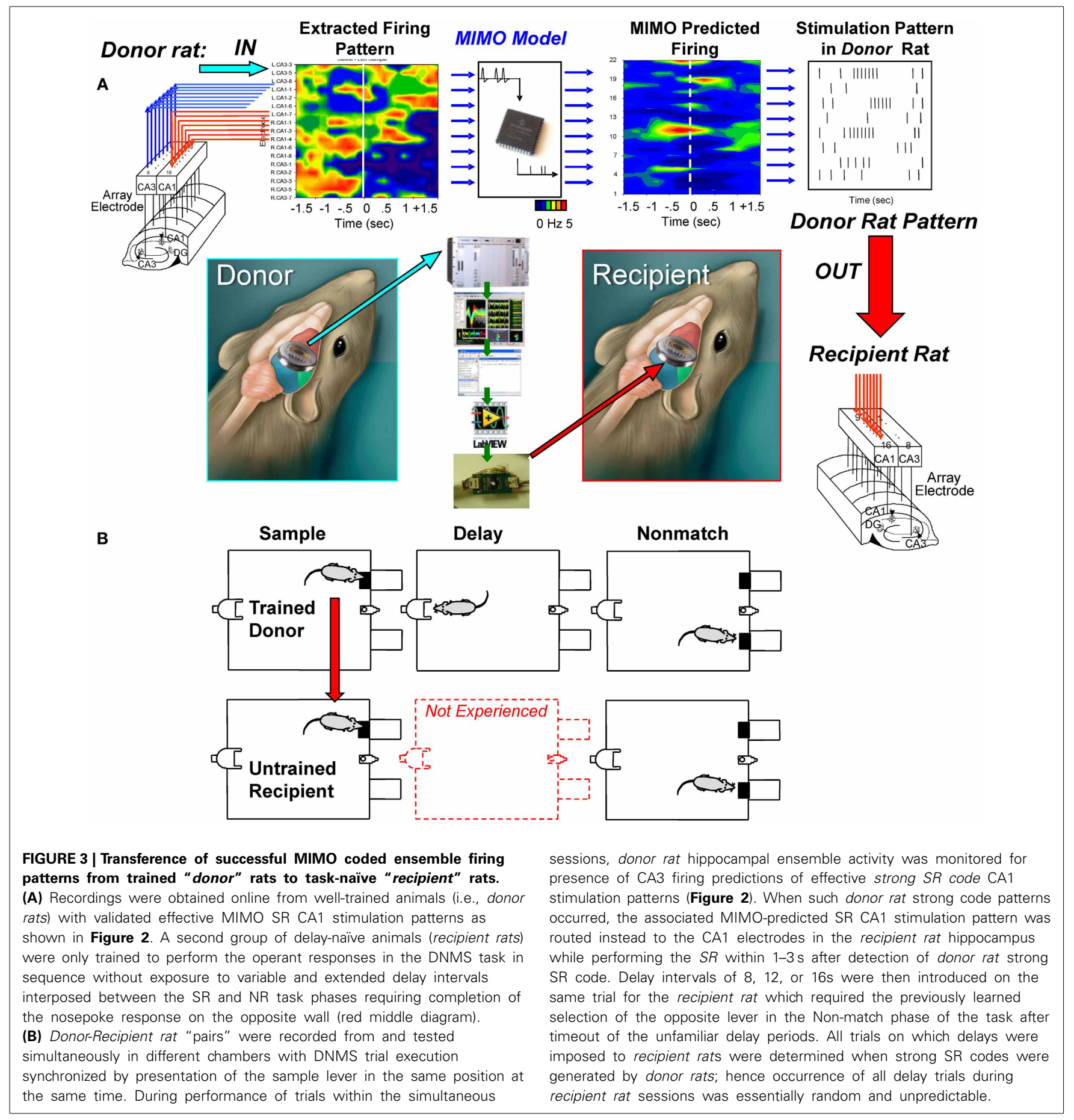

by delivery of MIMO SR CA1 stimulation and also the type of changes that occur under normal conditions related to improved vs. impaired performance in the same subjects. The influence of administered MIMO CA1 stimulation on synaptic connectivity during the session was assessed using local field potentials (LFPs) recorded from each of the 8 CA1 locations generated by stimulating a single electrode location in CA3 on the same array (Figure 6A) with min-to-max voltage ranges. For these LFPs, it was possible to assess changes in identified voltage LFP components related to excitatory and inhibitory synaptic inputs
(Hampson et al., 1989; Truccolo et al., 2002; Leung, 2011). CA1 LFPs were assessed before and after sessions in which MIMO stimulation was delivered and facilitated performance vs. sessions in which no stimulation was delivered. The most effective method of assessing such changes was to characterize differences in LFP waveforms by subtracting pre-session LFPs from post-session LFPs and comparing voltages (Post-Pre diff $_{\text {) }}$ in $10 \mathrm{~ms}$ segments as shown in Figure 6B. The resulting Post-Pre diff $_{\text {waveforms reflect }}$ changes in particular components of the LFP generated from the same CA3 stimulation location related to both excitatory and 


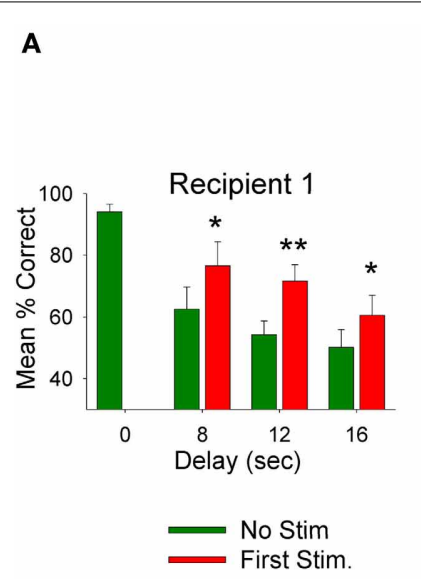

B

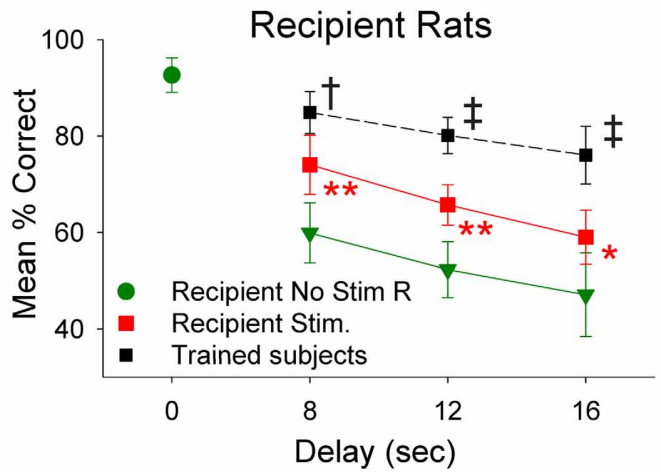

FIGURE 4 | Recipient rat performance on DNMS trials with unfamiliar superimposed delays facilitated by donor rat mediated SR

stimulation. (A) Individual DNMS performance of five different recipient rats subjected to trials with 8,12 , and $16 \mathrm{~s}$ delays shown for trials in which no stimulation was delivered (No Stim) or on trials on which a simultaneously paired donor rat delivery of MIMO SR CA1 stimulation pattern was delivered (Stim). The similarity across each graph indicates generality of facilitated performance on imposed delay trials with delivery of donor rat MIMO generated CA1 SR stimulation. Asterisks $* 0<0.01$, ${ }^{* *} p<0.001$, Stim donor rat vs. No Stim. (B) Overall performance of recipient rats $(n=5)$ is shown as mean ( \pm s.e.m.) \% correct trials with no delays (green dot-0.0s values) in comparison to trials with variable delays $(8,12,16 \mathrm{~s})$ without donor rat stimulation (green triangles-No Stim) delivered during the trial $\left[F_{(3,279)}=3.61, p<0.001\right]$; and performance on trials with the same delays but including donor rat MIMO strong SR code stimulation (Recipient Stim, red squares) which significantly improved performance compared to No Stim trials $\left[F_{(1,279)}=9.82, p<0.001\right]$. For comparison a plot of the average performance level of rats fully trained ( $n=20$ ) on the task at the same delays (Trained subjects) is shown (black squares) for comparison to recipient rat performance on stimulated trials $\left[F_{(1,1349)}=13.48, p=0.001\right.$, Trained subjects $>$ recipient rats $]$. Symbols: ${ }^{*} p<0.01,{ }^{* *} p<0.001,{ }^{\dagger} p<0.01,{ }^{\star} p=0.001$. inhibitory input over the same range of voltages, before and after behavioral sessions in which SR stimulation was, or was not, delivered.

Figure 6B shows the average of Post-Pre diff $_{\text {LFP waveforms for }}$ a single trained animal following SR stimulation (blue) vs. nonstimulation (red) sessions. It is clear that the LFP changes were related directly to both excitatory (40-80 ms) and inhibitory (90$175 \mathrm{~ms}$ ) components of well-characterized LFPs recorded from the cell layer at each of the eight CA1 locations (Leung, 2011).

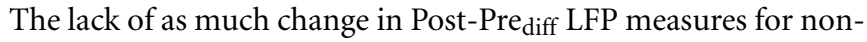
stimulation (red curve) vs. SR stimulation (blue curve) sessions in Figure 6B reflects the increase in excitatory synaptic input to the same CA1 locations that received strong code SR CA1 stimulation patterns and facilitated performance during the behavioral sessions in the same animal. To demonstrate a more general feature of this effect, the dotted curve in Figure 6B reflects a further difference of the LFP Post-Pre diff $_{\text {values in terms of subtracted }}$ Stim session Post-Pre diff $_{\text {LFP }}$ waveforms from similar Non-stim session waveforms, i.e., Diff stim $_{-}$Diff $_{\text {non-stim }}$ LFP values. Thus, the dotted curve in Figure 6B reflects the average differences in LFPs across 5 individual animals calculated in the same man-

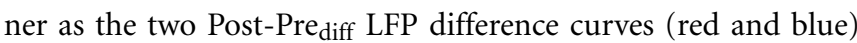

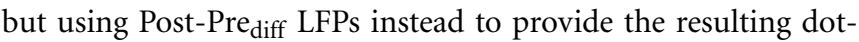
ted curve average difference of LFP waveform for SR stim vs. non-stim sessions (i.e., Diff stim $_{-}$Diff $\left._{\text {non-stim }}\right)$. Since this average difference (Diff stim - Diff $\left._{\text {non }- \text { stim }}\right)$ across all animals $(n=5)$ reflects alteration in the same LFP components as shown for individual waveforms in a single animal (red and blue Post-Pre diff $_{\text {f }}$ LFPs), it is clear that synaptic processes mediating CA3-to-CA1 transmission were increased by MIMO stimulation delivery during the DNMS sessions in which performance within and between animals was facilitated (Figures 4, 5). 

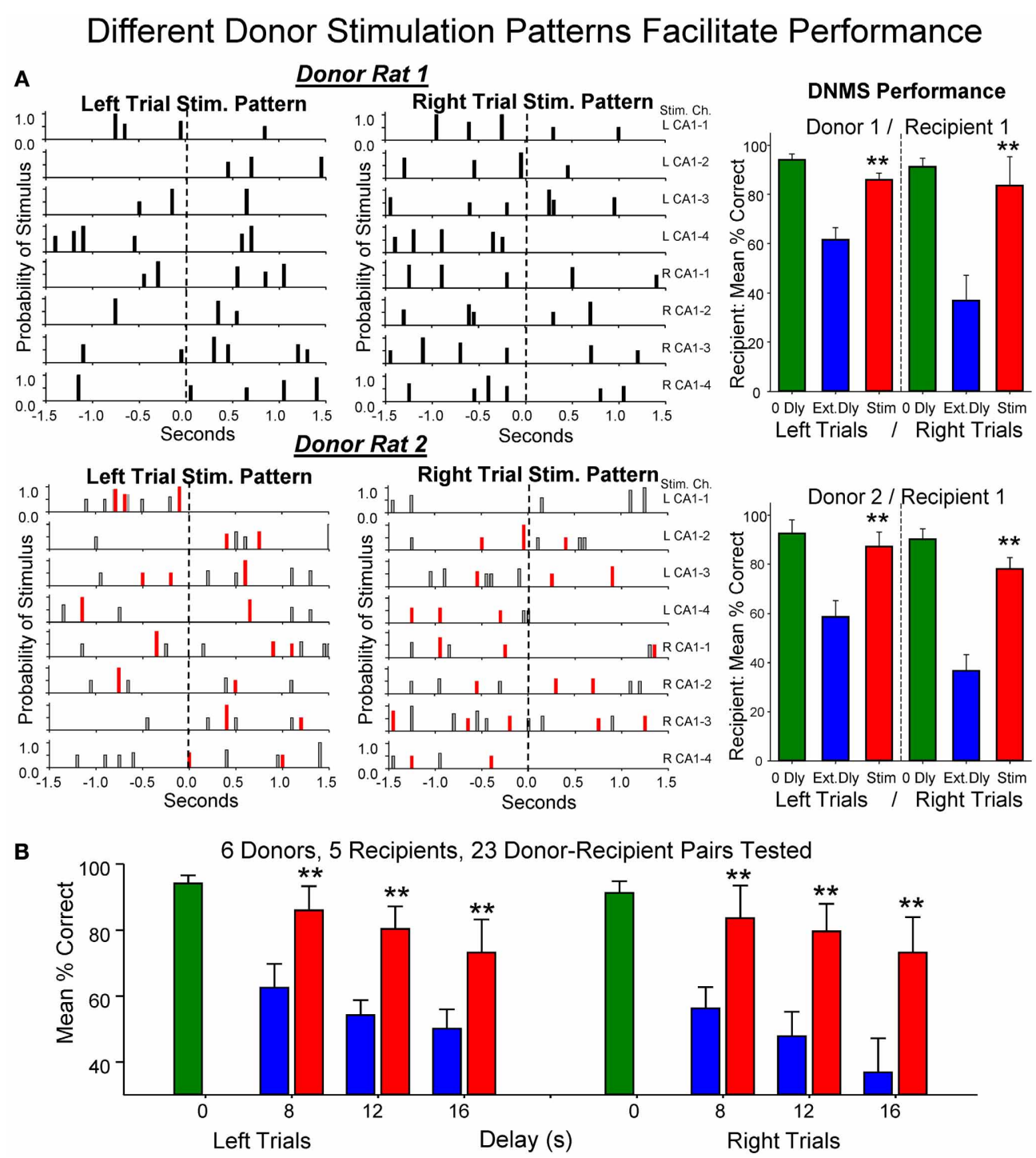

FIGURE 5 | Enhancement of performance of the same recipient rat by different donor rats. (A) Left: Delivered MIMO SR CA1 stimulation patterns showed for left and right lever trials from Donor rat 1 (upper) and Donor rat 2 (lower). Red marks in Donor rat 2 patterns reflect occurrences of identical pulses delivered in Donor rat 1 pattern (above) for direct comparison of the two SR Stimulation patterns delivered to the same Recipient rat on different trials. Right: Overall performance of the same Recipient rat for sessions in which SR Stimulation (Stim) on delay trials was contributed by Donor rat 1 (upper) and Donor rat 2 (lower) for left and right Sample lever trials summed over all delays (red) compared with delay trials in which SR stimulation was not delivered (blue). Green bars represent performance by the same Recipient rat on trials with no delay (0 Dly) presented in the same sessions as described above. (B) Lower plot shows overall average performance for all Donor/Recipient sessions ( $n=23$ ) for trials with Left and Right SR position and those which received donor rat SR stimulation (red) vs. no stimulation trials (blue) as a function of delay $(0,8,12,16 \mathrm{~s})$. Plots include all Donor/Recipient pairs, 5 different recipient rats paired with one or more donor rats $(n=6)$. Asterisks $\left({ }^{* *} p<0.001\right)$ indicate significant difference compared to trials with no donor rat stimulation (No Stim).

\section{DISCUSSION}

\section{DONOR/RECIPIENT RECOVERY OF HIPPOCAMPAL MEMORY: A MODEL} FOR APPLICATION TO MEMORY DEFICITS

The above findings provide highly significant evidence that functional working memory can be enhanced by delivery of donor rat MIMO CA1 patterned electrical stimulation to the CA1 field in the hippocampus of recipient rats. This shows that information encoded by individual neural events in naïve recipient rats can be effectively altered by substitution of donor rat MIMO derived electrical stimulus patterns in the same manner as demonstrated in prior studies in which effective stimulus trains were generated by, and delivered to, the same animal (Berger et al., 2011; Hampson et al., 2012a). The demonstration of improved task performance in naïve recipient rats (Figures 4, 5), verifies that donor rat SR CA1 stimulation was capable of inducing the type of encoding process necessary when facilitated retention of information was required because of interposed unfamiliar delays of variable duration (Figure 6). The fact that MIMO stimulation 


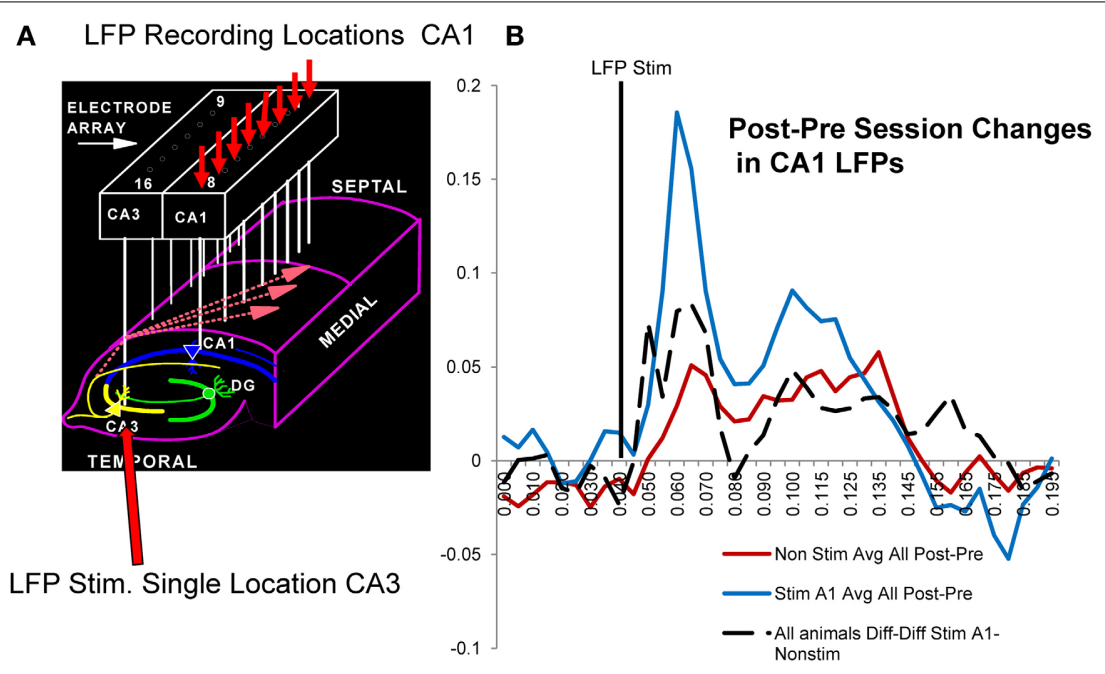

FIGURE 6 | Possible synaptic basis for facilitative Donor/Recipient MIMO SR stimulation. (A) Illustration of hippocampal synaptic connections between CA3 and CA1 cells in the same hippocampal region occupied by the same electrode array used to deliver SR Stimulation (Figures 1-3). Arrows show divergent projections from a single CA3 cell to multiple CA1 cells via Shaffer collateral connections used to determine changes in CA1 (small red arrows) local field potentials (LFPs) elicited by stimulation delivered to a single CA3 locus (large red arrow) before (Pre) and after (Post) behavioral sessions with MIMO SR Stimulation vs. non-stimulated sessions. (B) Average CA1 LFPs elicited by CA3 stimulation are plotted as differences (Post-Pre $\left.{ }_{\text {diff }}\right)$ in voltage amplitude measured at the indicated time points $(10 \mathrm{~ms})$ of the LFP after stimulus pulse delivery (vertical black line). Red:

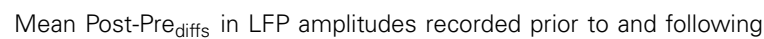
non-stimulation sessions for trained animals. Blue: Average Post-Pre ${ }_{\text {diffs }}$ in LFP amplitude following sessions in which SR stimulation was delivered to facilitate performance. Positive Post-Pre diffs $_{\text {reflect average voltage changes }}$ related to increased CA1 LFP components after the behavioral session relative to voltages elicited by the same CA3 current intensities prior to the session. These Post-Pre diffs for sessions in which SR stimulation facilitated task performance (blue curve) are shown compared to Post-Pre diffs $_{\text {for those }}$ sessions in which stimulation was not delivered (red curve). Dotted: Diff-Diff shows average difference between CA1 LFP Post-Pre diffs $_{\text {for }}$ SR stim vs. non-stim sessions (Stim-Non-stim) measured in 6 of the Donor rats (all animals A1-non-stim). can approximate normal ensemble firing involved in the encoding and retrieval of task-relevant information is consistent with other recent findings investigating relationships between multineuron firing in cortical ensembles and behavioral task requirements (Ross and Eichenbaum, 2006; Komorowski et al., 2009; Smith et al., 2009; Rouse et al., 2011). However, the demonstration that the patterns marked as effective and generated online in one animal, could be transferred via temporally matched electrical stimulation of similar CA1 regions in naïve recipient animals exposed to the same task contingencies, has not been shown previously. Although consistent in some ways with a recent "brainto-brain transfer" experiment (Pais-Viera et al., 2013) in which sensorimotor cortical signals were used to influence behavioral choice in the recipient rodent, the results presented here differ significantly because in that experiment, stimulation was delivered at the time of the behavioral response, whereas in our study, the stimulation corresponded to the encoding phase of the task (SR) and was delivered up to $16 \mathrm{~s}$ prior to the behavioral response, confirming transfer of a memory code, and not simply induction of a motor response. In addition, the lack of enhancement or transfer when several control procedures were employed in the above memory transfer paradigm; i.e., temporal relation to SR, reduced stimulation intensity, closed-loop dependence, etc. (Figures 1-5), strongly supports the specificity of the transference of donor rat MIMO model derived SR information for hippocampal function to naïve recipient rats for task-relevant performance.

These results provide important insight for extending donor/recipient procedures to functions performed by other brain regions and other behavioral endpoints as shown recently (PaisViera et al., 2013), and eventually to similar circumstances involving humans (Boettiger and D'Esposito, 2005; Smith et al., 2009; Hasson et al., 2012). Once fabricated into a neural prosthesis for recipients this unique technology could (1) immediately enhance task-specific performance, (2) repair damaged or impaired task-dependent brain circuitry, and possibly even, (3) provide neural encoding of task-relevant information without prior training. The long history of investigation with hippocampal recording in the behavioral context employed, and prior collaboration perfecting application of the MIMO model to these recordings (Hampson et al., 2008, 2012a; Berger et al., 2012; Marmarelis et al., 2013; Song et al., 2013), as well as recent applications to the non-human primate hippocampus (Hampson et al., 2013) and prefrontal cortex (Hampson et al., 2012c; Opris et al., 2012), provided the insight necessary to extrapolate how donor/recipient memory transference could occur as demonstrated here. However, the fact that transferred patterns of electrical brain stimulation have significant functional impact and are capable of modifying performance via strategic online delivery provides another demonstration of donor-recipient brain compatibility (Pais-Viera et al., 2013), but this application to hippocampus for improving memory is the first demonstration specific to brain cognitive function. Such results not only provide important new insight into how hippocampal circuits can be operated to process memory-dependent information via external control, but also provide a basis for extending and/or perfecting similar donor/recipient type devices (Jarosiewicz et al., 2008; 
Venkatraman and Carmena, 2011; Hasson et al., 2012) to enhance and/or replace memory deficiencies in humans.

\section{ACKNOWLEDGMENTS}

The authors appreciate the efforts of Vernell Collins, Rodrigo Espana, Lihong Shi, Chad Collins, George McLeod, Anushka Goonawardena. This work was supported by contracts to Sam A. Deadwyler and Theodore W. Berger from the DARPA REMIND Program (N66001-C-09-2080 and N66001-C-09-2081, COL. Geoff Ling, Program Manager), NSF EEC-0310723 (to Theodore W. Berger), NIDA DA07625 (to Sam A. Deadwyler).

\section{SUPPLEMENTARY MATERIAL}

The Supplementary Material for this article can be found online at: http://www.frontiersin.org/journal/10.3389/fnsys.2013. 00120/abstract

\section{REFERENCES}

Berger, T. W., Hampson, R. E., Song, D., Goonawardena, A., Marmarelis, V. Z., and Deadwyler, S. A. (2011). A cortical neural prosthesis for restoring and enhancing memory. J. Neural Eng. 8, 046017. doi: 10.1088/1741-2560/8/4/ 046017

Berger, T. W., Song, D., Chan, R. H. M., Marmarelis, V. Z., LaCoss, J., Wills, J., et al. (2012). A hippocampal cognitive prosthesis: multi-input, multi-output nonlinear modeling and VLSI implementation. Trans. Neural. Syst. Rehabil. Eng. 20, 198-211. doi: 10.1109/TNSRE.2012.2189133

Boettiger, C. A., and D'Esposito, M. (2005). Frontal networks for learning and executing arbitrary stimulus-response associations. J. Neurosci. 25, 2723. doi: 10.1523/JNEUROSCI.3697-04.2005

Deadwyler, S. A., and Hampson, R. E. (1997). "The significance of neural ensemble codes during behavior and cognition," in Annual Review of Neuroscience, Vol. 20, eds W. M. Cowan, E. M. Shooter, C. F. Stevens, and R. F. Thompson (Palo Alto, CA: Annual Reviews, Inc), 217-244. doi: 10.1146/annurev.neuro. 20.1.217

Deadwyler, S. A. and Hampson, R. E. (2006). Temporal coupling between subicular and hippocampal neurons underlies retention of trial-specific events. Behav. Brain Res. 174, 272-280. doi: 10.1016/j.bbr.2006.05.038

Deadwyler, S. A., Goonawardena, A. V., and Hampson, R. E. (2007). Short-term memory is modulated by the spontaneous release of endocannabinoids: evidence from hippocampal population codes. Behav. Pharm. 18, 571-580. doi: 10.1097/FBP.0b013e3282ee2adb

Eichenbaum, H., and Fortin, N. J. (2009). The neurobiology of memory based predictions. Philos. Trans. R. Soc. Lond. B Biol. Sci. 364, 1183. doi: 10.1098/rstb.2008.0306

Hasson, U., Ghazanfar, A. A., Galantucci, B., Garrod, S., and Keysers, C. (2012). Brain-to-brain coupling: a mechanism for creating and sharing a social world. Trends Cogn. Sci. 16, 114-121. doi: 10.1016/j.tics.2011. 12.007

Hampson, R. E., Foster, T. C., and Deadwyler, S. A. (1989). Effects of delta9-tetrahydrocannabinol on sensory evoked hippocampal activity in the rat: principal components analysis and sequential dependency. J. Pharmacol. Exp. Ther. 251, 870-877.

Hampson, R. E., Jarrard, L. E., and Deadwyler, S. A. (1999). Effects of ibotenate hippocampal and extrahippocampal destruction on delayedmatch and -nonmatch-to-sample behavior in rats. J. Neurosci. 19, 1492-1507.

Hampson, R. E., Simeral, J. D., and Deadwyler, S. A. (2008). "Neural population recording in behaving animals: constituents of the neural code for behavior," in Neural Population Encoding, eds C. R Holscher and M. H. Munk (Cambridge: Cambridge University Press), 111-128.

Hampson, R. E., Sweatt, A. J., Goonawardena, A. V., Song, D., Chan, R. H., Marmarelis, V. Z., et al. (2011). Memory encoding in hippocampal ensembles is negatively influenced by cannabinoid CB1 receptors. Behav. Pharmacol. 22, 335-346. doi: 10.1097/FBP.0b013e3283473bfd
Hampson, R. E., Song, D., Chan, R. H. M., Sweatt, A. J., Fuqua, J., Gerhardt, G. A., et al. (2012a). A nonlinear model for hippocampal cognitive prostheses: memory facilitation by hippocampal ensemble stimulation. Trans. Neural Syst. Rehabil. Eng. 20,184-197. doi: 10.1109/TNSRE.2012.2189163

Hampson, R. E., Song, D., Chan, R. H. M., Sweatt, A. J., Riley, M. R., Goonawardena, A. V., et al. (2012b). Closing the loop for memory prostheses: detecting the role of hippocampal neural ensembles using nonlinear models. Trans. Neural Syst. Rehabil. Eng. 20, 510-525. doi: 10.1109/TNSRE.2012.2190942

Hampson, R. E., Gerhardt, G. A., Marmarelis, V. Z., Song, D., Opris, I., Santos, L., et al. (2012c). Facilitation and restoration of cognitive function in primate prefrontal cortex by a neuroprosthesis that utilizes minicolumnspecific neural firing. J. Neural Eng. 9, 056012. doi: 10.1088/1741-2560/9/5/ 056012

Hampson, R. E., Song, D., Opris, I., Santos, L. M., Gerhardt, G. A., Marmarelis, V. Z., et al. (2013). Facilitation of memory encoding in primate hippocampus by a neuroprosthesis that promotes task specific neural firing. J. Neural Eng. 10:066013. doi: 10.1088/1741-2560/10/6/066013

Jarosiewicz, B., Chase, S. M., Fraser, G. W., Velliste, M., Kass, R. E., and Schwartz, A. B. (2008). Functional network reorganization during learning in a braincomputer interface paradigm. Proc. Natl. Acad. Sci. U.S.A. 105, 19486. doi: 10.1073/pnas.0808113105

Komorowski, R. W., Manns, J. R., and Eichenbaum, H. (2009). Robust conjunctive item-place coding by hippocampal neurons parallels learning what happens where. J. Neurosci. 29, 9918. doi: 10.1523/JNEUROSCI.137809.2009

Leung, S. L. (2011). "Field potential generation and current source density analysis," in Electrophysiological Recording Techniques, eds R. P. Vertes and R. W. Stackman (New York, NY: Humana Press), 1-25. doi: 10.1007/978-1-60327202-5_1

Marmarelis, V. Z. (2004). Nonlinear Dynamic Modeling of Physiological Systems. Hoboken, NJ: Wiley-IEEE Press. doi: 10.1002/9780471679370

Marmarelis, V. Z., Shin, D. C., Song, D., Hampson, R. E., Deadwyler, S. A., and Berger, T. W. (2013). Nonlinear modeling of dynamic interactions within neuronal ensembles using principal dynamic modes. J. Comput. Neurosci. 34, 73-87. doi: 10.1007/s10827-012-0407-7

Opris, I., Hampson, R. E., Gerhardt, G. A., Berger, T. W., and Deadwyler, S. A. (2012). Columnar processing in primate prefrontal cortex: evidence for executive control microcircuits. J. Cogn. Neurosci. 24, 2334-2347. doi: 10.1162/jocn_a_00307

Pais-Viera, M., Lebedev, M., Kunicki, C., Wang, J., and Nicolelis, M. A. L. (2013). A brain-to-brain interface for real-time sensorimotor information. Sci. Rep. 3, 1319. doi: $10.1038 /$ srep01319

Ross, R. S., and Eichenbaum, H. (2006). Dynamics of hippocampal and cortical activation during consolidation of a nonspatial memory. J. Neurosci. 26, 4852. doi: 10.1523/JNEUROSCI.0659-06.2006

Rouse, A. G., Stanslaski, S. R., Cong, P., Jensen, R. M., Afshar, P., Ullestad, D., et al. (2011). A chronic generalized bi-directional brain-machine interface. J. Neural Eng. 8, 36018. doi: 10.1088/1741-2560/8/3/036018

Smith, N. J., Narayanan, N. S., and Laubach, M. (2009). Past performance is indicative of future returns. Neuron 63, 146. doi: 10.1016/j.neuron. 2009.07.007

Song, D., Chan, R. H., Marmarelis, V. Z., Hampson, R. E., Deadwyler, S. A., and Berger, T. W. (2009). Nonlinear modeling of neural population dynamics for hippocampal prostheses. Neural Netw. 22, 1340-1351. doi: 10.1016/j.neunet.2009.05.004

Song, D., Wang, H., Tu, C. Y., Marmarelis, V. Z., Hampson, R. E., Deadwyler, S. A., et al. (2013). Identification of sparse neural functional connectivity using penalized likelihood estimation and basis functions. J. Comput. Neurosci. 35, 335-357. doi: 10.1007/s10827-013-0455-7

Truccolo, W. A., Ding, M., Knuth, K. H., Nakamura, R., and Bressler, S. L. (2002). Trial-totrial variability of cortical evoked responses: implications for the analysis of functional connectivity. Clin. Neurophysiol. 113, 206-226. doi: 10.1016/S1388-2457(01)00739-8

Venkatraman, S., and Carmena, J. M. (2011). Active sensing of target location encoded by cortical microstimulation. IEEE Trans. Neural Syst. Rehabil. Eng. 19, 317-324. doi: 10.1109/TNSRE.2011.2117441

Witter, M. P., and Amaral, D. G., (2004). "Hippocampal formation," in The Rat Nervous System, ed G. Paxinos (San Diego, CA: Academic Press), 635-704. 
Zanos, T. P., Courellis, S. H., Berger, T. W., Hampson, R. E., Deadwyler, S. A., and Marmarelis, V. Z. (2008). Nonlinear modeling of causal interrelationships in neuronal ensembles. IEEE Trans. Neural Syst. Rehabil. Eng. 16, 336-352. doi: 10.1109/TNSRE.2008.926716

Conflict of Interest Statement: The authors declare that the research was conducted in the absence of any commercial or financial relationships that could be construed as a potential conflict of interest.

Received: 11 October 2013; accepted: 06 December 2013; published online: 26 December 2013.
Citation: Deadwyler SA, Berger TW, Sweatt AJ, Song D, Chan RHM, Opris I, Gerhardt GA, Marmarelis VZ and Hampson RE (2013) Donor/recipient enhancement of memory in rat hippocampus. Front. Syst. Neurosci. 7:120. doi: 10.3389/fnsys. 2013.00120

This article was submitted to the journal Frontiers in Systems Neuroscience.

Copyright (C) 2013 Deadwyler, Berger, Sweatt, Song, Chan, Opris, Gerhardt, Marmarelis and Hampson. This is an open-access article distributed under the terms of the Creative Commons Attribution License (CC BY). The use, distribution or reproduction in other forums is permitted, provided the original author(s) or licensor are credited and that the original publication in this journal is cited, in accordance with accepted academic practice. No use, distribution or reproduction is permitted which does not comply with these terms. 